\title{
Enhancing the role of doctors in preventing suicide
}

DOI: $10.12809 /$ hkmj134122
The aftermath of suicide is devastating. At a personal level, it is often painfully experienced by the survivors who are emotionally tied to the victim of suicide directly. It is well known that $90 \%$ of suicide deaths are by people suffering from mental illness or substance abuse, problems for which a vast majority of such individuals are untreated. ${ }^{1}$ A substantial proportion of people with psychological sufferings do not seek medical and psychosocial treatments until the enduring health issues and related intricate psychosocial factors culminate to cause unbearable psychological pain that the irreversible outcome of death by suicide becomes unveiled.

In 2012, the World Health Organization's Health Evidence Network (WHO HEN) published a review of 34 types of suicide prevention interventions, which were abstracted in the form of published systematic reviews. ${ }^{2}$ These included public health preventive efforts for the general population (universal intervention), targeted interventions focused on highrisk populations showing at-risk behaviour (selective intervention) as well as post-ventions after attempted suicide (indicated intervention). ${ }^{2}$ The report summarised the promising effectiveness of certain intervention, namely: (i) universal interventions such as school-based suicide prevention programmes enhancing coping skills and social support for at-risk students. These showed beneficial effects on intermediate outcomes (such as suicide tendencies and risk factors for suicide), while restricting access to lethal means could reduce cause-specific but not overall suicide rates; and (ii) targeted interventions such as psychosocial and pharmacological treatments were shown to be effective in reducing rates of repeated self-harm in indicated situations such as those who made a recent suicide attempt or with pre-existing mood disorders. The WHO HEN's recent report complements the findings from an earlier systematic review on suicide prevention strategies by Mann et al. ${ }^{3}$ This report alluded to the roles of physician education in recognising and treating depression, as well as restricting access to lethal methods, while others entailed public education, screening programmes, and media education for which more evidence of efficacy was needed. ${ }^{3}$ Convergent views from both reviews suggest potentially important roles of physicians.

Generalising the summary findings must be made with caution, due to different sociocultural/ clinical settings and by virtue of the methodological heterogeneity. Nonetheless, these findings guide medical professionals to rethink their roles in suicide prevention in a systematic way, as well as increasing awareness of evidence-based practice of suicide prevention and post-vention efforts in a multi-level model that has potential implications for collaborative care.

Primary care and specialist settings are important help-seeking avenues for the at-risk clinical population, as evidence suggests that over $45 \%$ of people who died of suicide had contacts with mental health professionals and primary health care providers in the 4 weeks prior to suicide. ${ }^{4}$ A local psychoautopsy study found that over $70 \%$ of older adults had consulted primary care physicians in the month before committing suicide. ${ }^{5}$ Physicians have privileged roles in assessing at-risk individuals in terms of biopsychosocial problems. Via this unique role, potentially they can engage the individual in a collaborative care plan to modify the volatile risk state. This in turn may leverage a person to activate resources to surpass the existential crisis meaningfully. What are the challenges faced by physicians who might have played crucial roles in preventing these premature deaths?

Yousuf et al's paper ${ }^{6}$ in this issue of the Hong Kong Medical Journal shared a Hong Kong experience of implementing an undergraduate suicide prevention module in a local medical school. The study forms the empirical basis for a timely reflection of resistance to detecting and managing peri-suicidal states or their underlying risk factors among medical and other allied health professionals in Hong Kong. Undergraduate medical curricula in Hong Kong are similar to those of UK and Australia in many ways. Systematic surveys of undergraduate medical curricula in UK and Australia have shown that typically they deliver very little knowledge and skill in suicide prevention, and the mode of such teaching is usually lecture-based. ${ }^{7,8}$ Notably, in the Australian study by Hawgood et al, students who previously received suicide prevention education rated themselves significantly higher in terms of skills-based suicide prevention abilities than those who had not. Moreover, most of them (including those who received limited skills-based training) rated these capabilities very high in importance for the medical profession. ${ }^{8}$ The willingness to receive suicide prevention training and the emphasis on skills rather than mere knowledge emphasises the critical potential of developing a value-driven medical curriculum. Yousuf et al's study in this issue ${ }^{6}$ alluded to the malleability of undergraduates' attitudes to suicide prevention, which was attained via a dedicated undergraduate module that was highly valued and well received by Hong Kong medical students. 
Such a model of skill acquisition for physicians-intraining may even be extended to postgraduate and continuous education on suicide prevention.

While clinical skills and knowledge are the main determinants of one's clinical practice and adherence to best evidence-based treatment, there are nonclinical factors (such as physician time constraints, available health resources, organisational factors like size and types of clinical practice) that may compromise optimal practice. ${ }^{9}$ In the area of suicide prevention, multi-level collaboration, dynamic appraisal of immediate and remote risks, as well as a personalised care programme constitute the real life challenges for a doctor. For this goal, physicians must take an active role in suicide prevention, and overcome professional pessimism especially among those not working in a collaborative care setting specialising in mental health problems. Doctors may hesitate to uncover such problems when they perceive there is no immediately available professional peer back-up or there is no guaranteed safe outcome, given the fluidity of biopsychosocial risk factors. Nonetheless, some research shows that educating primary care clinicians can help protect against suicide, primarily by improving the recognition of depression and leading to the increased prescribing of antidepressants. ${ }^{10-12}$ These effects are stronger when collaborative care models of depression treatment are instituted. ${ }^{13-15}$
Although the relative risk of suicide among doctors may not be exceptional compared to other occupational groups, they are often able to elude clinical detection as they often prefer to medicate themselves instead of seeking outside medical help. ${ }^{16}$ Thus, acquiring suicide prevention skills by physicians may result in self-directed benefit through fostering self-empathy and a help-seeking culture in medical professionals.

The bottom-up approach to enhancing role of doctors in suicide prevention can begin with undergraduate education, and continue into postgraduate training. The fundamental changes and consolidation in psychological readiness, clinical skills, and knowledge are likely the first steps to any sustainable cross-disciplinary collaborative care model, which could operate in tertiary care, primary care, or community interfaces.

\footnotetext{
Sandra Chan, FHKCPsych, FHKAM (Psychiatry)

Email: schan@cuhk.edu.hk

Linda Lam, MD, FHKAM (Psychiatry)

Helen Chiu, FHKCPsych, FHKAM (Psychiatry)

Department of Psychiatry

The Chinese University of Hong Kong

Shatin

Hong Kong
}

\section{References}

1. Cavanagh JT, Carson AJ, Sharpe M, Lawrie SM. Psychological autopsy studies of suicide: a systematic review. Psychol Med 2003;33:395-405. cross ref

2. Scott A, Guo B. World Health Organization's Health Evidence Network. Geneva: World Health Organization; Jul 2012.

3. Mann JJ, Apter A, Bertolote J, et al. Suicide prevention strategies: a systematic review. JAMA 2005;294:2064-74. cross ref

4. Luoma JB, Martin CE, Pearson JL. Contact with mental health and primary care providers before suicide: a review of the evidence. Am J Psychiatry 2002;159:909-16. cross ref

5. Chiu HF, Yip PS, Chi I, et al. Elderly suicide in Hong Kong-a case-controlled psychological autopsy study. Acta Psychiatr Scand 2004;109:299-305. cross ref

6. Yousuf S, Beh PS, Wong PW. Attitudes towards suicide following an undergraduate suicide prevention module: experience of medical students in Hong Kong. Hong Kong Med J 2013;19:377-85.

7. Karim K, Edwards R, Dogra N, et al. A survey of the teaching and assessment of undergraduate psychiatry in the medical schools of the United Kingdom and Ireland. Med Teach 2009;31:1024-9. cross ref

8. Hawgood JL, Krysinska KE, Ide N, De Leo D. Is suicide prevention properly taught in medical schools? Med Teach 2008;30:28795. cross ref

9. Hajjaj FM, Salek MS, Basra MK, Finlay AY. Non-clinical influences on clinical decision-making: a major challenge to evidencebased practice. J R Soc Med 2010;103:178-87. cross ref

10. Rihmer Z, Belsö N, Kalmár S. Antidepressants and suicide prevention in Hungary. Acta Psychiatr Scand 2001;103:238-9.

11. Rutz W, von Knorring L, Wålinder J. Frequency of suicide on Gotland after systematic postgraduate education of general practitioners. Acta Psychiatr Scand 1989;80:151-4. cross ref

12. Rutz W, von Knorring L, Wålinder J. Long-term effects of an educational program for general practitioners given by the Swedish Committee for the Prevention and Treatment of Depression. Acta Psychiatr Scand 1992;85:83-8. cross ref

13. Szanto K, Kalmar S, Hendin H, Rihmer Z, Mann JJ. A suicide prevention program in a region with a very high suicide rate. Arch Gen Psychiatry 2007;64:914-20. cross ref

14. Alexopoulos GS, Reynolds CF 3rd, Bruce ML, et al. Reducing suicidal ideation and depression in older primary care patients: 24-month outcomes of the PROSPECT study. Am J Psychiatry 2009;166:882-90. cross ref

15. Gilbody S, Bower P, Fletcher J, Richards D, Sutton AJ. Collaborative care for depression: a cumulative meta-analysis and review of longer-term outcomes. Arch Intern Med 2006;166:2314-21. cross ref

16. Hawton K, Clements A, Simkin S, Malmberg A. Doctors who kill themselves: a study of the methods for suicide. QJM 2000;93:351-7. cross ref 